\section{PERCEPÇÕES E VIVÊNCIAS DE CUIDADORES FAMILIARES DE IDOSOS ACAMADOS}

\author{
Perceptions and experiences of family caregivers of bedridden \\ elderly
}

\author{
Percepciones y vivencias de cuidadores familiares de mayores \\ encamados
}

Artigo Original

\title{
RESUMO
}

Objetivo: Conhecer as percepções e vivências de cuidadores familiares de idosos acamados. Métodos: Estudo descritivo, exploratório, com abordagem qualitativa, realizado em janeiro de 2015 com quatro cuidadores em uma Unidade de Saúde da Família no município de Araripe-CE. A coleta de dados deu-se a partir de uma entrevista semiestruturada, sendo as informações organizadas através da técnica de análise de conteúdo. A partir da análise dos depoimentos dos cuidadores, emergiram três categorias: o processo de dependência do idoso; dificuldades vivenciadas no cotidiano do cuidador; e satisfação com o serviço de saúde no domicílio. Resultados: O processo de dependência do idoso ocorreu como consequência de processos patológicos, como neoplasia, acidente vascular encefálico e demência. No entanto, notou-se também que os fenômenos fisiológicos, próprios da velhice, também podem levar o indivíduo a tornar-se dependente dos cuidadores. Quanto às dificuldades enfrentadas pelos cuidadores, estes relataram ausência de envolvimento maior da família, haja vista que a centralização do trabalho gera sobrecarga e, consequentemente, afeta o cuidado com o idoso. $\mathrm{O}$ trabalho em equipe no contexto domiciliar é fundamental, pois permite um atendimento complementar e integral para o binômio idoso/cuidador. Conclusão: Envelhecer de forma saudável é o grande desafio a ser superado, pois o estabelecimento de hábitos de vida saudáveis é uma dificuldade vivenciada por todos os seguimentos sociais. Faz-se cada vez mais necessária a melhoria do atendimento domiciliar, com vistas à prestação de suporte aos cuidadores com vistas à melhor qualidade de vida do idoso acamado, bem como do cuidador.

Descritores: Cuidadores; Idoso; Família; Idoso Fragilizado.

\section{ABSTRACT}

Objective: To know the perceptions and experiences of family caregivers of bedridden elderly. Methods: A qualitative descriptive exploratory study conducted in January 2015 with four caregivers in a Family Health Center in the municipality of Araripe, CE. Data were collected through semi-structured interviews and the information was organized using the content analysis technique. A total of three categories emerged from the analysis of the reports of caregivers: the dependence process of the elderly; daily difficulties experienced by the caregiver; and satisfaction with the home care service. Results: The dependence process of the elderly took place as a consequence of pathological processes such as neoplasm, cerebrovascular accident and dementia. However, it could also be observed that physiological phenomena - common in old age - can also make individuals dependent on caregivers. As to the difficulties faced by the caregivers, they reported the need for greater involvement by the family, given that the centralization of work generates an overload and hence affects the care of the elderly. Teamwork in the home care context is fundamental, given that it allows a complementary and comprehensive care to the elderly/caregiver binomial. Conclusion: Healthy aging is a major challenge to be overcome given that the development of a healthy lifestyle is difficult in all social strata. It is necessary to improve home care in order to provide support to caregivers so that the quality of life of bedridden elderly and caregivers is improved.

Descriptors: Caregivers; Elderly; Family; Frail Elderly.

\author{
Ticiano Magalhães Dantas ${ }^{(1,2)}$ \\ Lindaiane Bezerra Rodrigues ${ }^{(2,3)}$ \\ Wine Suélhi dos Santos ${ }^{(3)}$ \\ Josefa Cristina Dias ${ }^{(3)}$ \\ Nathalia Matos de Santana ${ }^{(3)}$ \\ Ivana Cristina Vieira de Lima ${ }^{(4)}$
}

1) Prefeitura Municipal de Araripe - Araripe

(CE) - Brasil

2) Universidade Regional do Cariri - URCA

- Crato (CE) - Brasil

3) Faculdade de Ciências Aplicadas Dr. Leão Sampaio - FALS - Juazeiro do Norte

(CE) - Brasil

4) Núcleo de Tecnologias e Educação em Saúde/Universidade Federal do Ceará -NUTEDS/UFC - Fortaleza (CE) - Brasil
Recebido: 08/04/2015

Revisado em: 20/05/2015 Aceito em: 09/09/2015 


\section{RESUMEN}

Objetivo: Conocer las percepciones y vivencias de cuidadores familiares de mayores encamados. Métodos: Estudio descriptivo, exploratorio de abordaje cuantitativo realizado en enero de 2015 con cuatro cuidadores de una Unidad de Salud de la Familia del municipio de Araripe-CE. La recogida de datos se dio a partir de una entrevista semiestructurada y las informaciones fueron organizadas a través de la técnica del Análisis de Contenido. A partir del análisis de los relatos de los cuidadores emergieron tres categorías: el proceso de dependencia del mayor; las dificultades vivenciadas en el cotidiano del cuidador; y la satisfacción con el servicio de salud del domicilio. Resultados: El proceso de dependencia del mayor se dio como consecuencia de procesos patológicos como la neoplasia, el accidente vascular encefálico y la demencia. Sin embargo, se notó también que los fenómenos fisiológicos, propios de la vejez, también pueden llevar el individuo a depender de los cuidadores. Sobre las dificultades afrontadas por los cuidadores los mismos relataron la ausencia de mayor envolvimiento de parte de la familia ya que la centralización del trabajo genera sobrecarga y, en consecuencia, afecta el cuidado con el mayor. El trabajo del equipo en el contexto domiciliario es fundamental pues permite una atención complementaria e integral del binomio mayor/cuidador. Conclusión: Envejecer de manera saludable es el gran desafio a superar pues el establecimiento de hábitos de vida saludables es una dificultad vivenciada por todos los seguimientos sociales. Se hace necesario cada vez más la mejoría de la atención domiciliaria para la prestación del apoyo a los cuidadores para una mejor calidad de vida del mayor encamado así como del cuidador.

Descriptores: Cuidadores; Anciano; Família; Anciano Frágil.

\section{INTRODUÇÃO}

Envelhecer é um processo natural e cronológico que engloba todos os indivíduos, porém, a idade cronológica não pode ser dita como fator determinante para diferenciar as pessoas idosas ${ }^{(1)}$. No processo de senilidade, o organismo sofre modificações naturais ${ }^{(2)}$.

Segundo o Instituto Brasileiro de Geografia e Estatística (IBGE), o Brasil é composto por 14,9 milhões de idosos, o que corresponde a $7,4 \%$ da população total. Estima-se que, em 2060, a população de pessoas idosas seja de 58,4 milhões. Nesse período, a expectativa média de vida do brasileiro deve aumentar dos atuais 75 anos para $81 \operatorname{anos}^{(3)}$.

Cada indivíduo envelhece ao seu tempo e ao seu ritmo, influenciado por elementos externos, genéticos e estilo de vida. Há dois fatores predominantes que atuam sobre o envelhecimento: são eles os fatores intrínsecos, que atuam a partir do interior da pessoa, descritos como envelhecimento normal; e os fatores extrínsecos, que são os agentes físicos (temperatura, radiação, umidade, força, produtos químicos) e infecciosos (vírus, fungos, bactérias, protozoários) ${ }^{(4)}$.

O envelhecimento constitui um avanço social, sendo a longevidade evidente nos tempos atuais. Contudo, consiste também em um problema de saúde pública, uma vez que estas pessoas necessitam de atendimento e suporte especializado, em decorrência da maior probabilidade de desenvolverem doenças, devido ao avanço da idade e às mudanças anatômicas e fisiológicas que podem levar à dependência ${ }^{(5)}$.

As modificações decorrentes desse processo podem afetar todo o organismo, mas alguns sistemas estão mais susceptíveis às complicações, como: sistema cardiovascular, sistema nervoso, sistema osteomuscular e sistema endócrino. Alterações com tendências a condições crônicas podem levar o idoso à perda da autonomia, à dependência $\mathrm{e}$ a alterações sociais e emocionais ${ }^{(6)}$.

Destacam-se alguns exemplos de alterações normais do envelhecimento, como a sarcopenia (redução da massa muscular), a osteopenia (redução da massa óssea), a redução do conteúdo de água corporal, a redução da capacidade aeróbica, dentre outros. Essas alterações propiciam o aumento do risco de acidentes, como as quedas, que podem levar o idoso a ficar restrito ao leito e trazer repercussões como o aparecimento de úlceras por pressão, infecções respiratórias e urinárias, dentre outras ${ }^{(6)}$.

Conforme a Constituição Federal datada de 1988, o idoso é visto como um indivíduo que necessita de proteção e cuidados, descrevendo que a família, a sociedade e o Estado têm o dever de apará-los e dar-lhes suporte para o convívio e bem-estar na comunidade ${ }^{(7)}$.

$\mathrm{O}$ ato de cuidar de idosos, às vezes, é assumido de forma repentina por algum membro da família, recaindo com maior frequência sobre o sexo feminino ${ }^{(8)}$. Tornarse cuidador é algo que requer atenção, responsabilidade, paciência e amor ao próximo. Porém, dentre esses requisitos, é necessário um suporte básico para atender às necessidades dos idosos, contudo, esse suporte por vezes é inexistente, tendo a família a responsabilidade de prestar cuidados aos idosos acamados, ainda que sem o conhecimento técnico necessário para tanto.

O presente trabalho teve seu enfoque na vivência do cuidador do idoso acamado, destacando os problemas enfrentados no ambiente domiciliar, bem como as dificuldades decorrentes da falta de suporte da atenção básica e o papel do apoio social. Nesse contexto, levantouse a seguinte questão: como os cuidadores familiares de idosos acamados percebem e vivenciam o processo de cuidar deles?

Assim, objetivou-se conhecer as percepções e vivências de cuidadores familiares com idosos acamados. 


\section{MÉTODOS}

Optou-se por um estudo do tipo descritivo e exploratório, com abordagem qualitativa.

$\mathrm{Na}$ pesquisa qualitativa, o conhecimento é originário de informações de pessoas diretamente vinculadas com a experiência estudada, portanto, não podem ser controladas e generalizadas. Os dados, por sua vez, não são coisas isoladas, acontecimentos fixos, percepções puras e definidas, de modo que todos os acontecimentos em um contexto são igualmente importantes, como a constância das manifestações, a sua ocasionalidade, a frequência, a interrupção, a fala e o silêncio ${ }^{(9)}$.

Por sua vez, a pesquisa exploratória permite acrescentar experiências acerca do caráter do tema ou do fenômeno através do envolvimento com o tema estudado. Portanto, a pesquisa exploratória é a que explora algo novo, que frequentemente não é considerado ainda ciência, mas serve de base à ciência. Como afirmam alguns autores, a pesquisa exploratória não elabora hipóteses, sendo utilizada quando se tem poucos estudos e conhecimentos científicos sobre o tema $^{(10)}$.

Já o caráter descritivo se dá a partir dos relatos feitos pelos sujeitos em estudo. As pesquisas descritivas objetivam a descrição das características de alguma população ou estabelece analogias entre variáveis ${ }^{(11)}$.

A pesquisa ocorreu no mês de janeiro de 2015, no município de Araripe-CE, localizado na microrregião da Chapada do Araripe, distando 526,8 Km da capital Fortaleza. Conta com uma população estimada de 21.214 habitantes $^{(12)}$.

Atualmente, o município de Araripe possui oito Unidades de Saúde da Família (USF) e oito Equipes de Saúde da Família (ESF), sendo três na zona urbana e cinco na zona rural, que oferecem os seguintes serviços: acolhimento, atendimento médico, odontológico e de enfermagem. Os serviços desenvolvem ações de promoção, proteção e recuperação da saúde. A rede secundária de saúde de Araripe conta também com um hospital de pequeno porte que atende às quatro clínicas básicas (médica, cirúrgica, obstétrica e pediátrica). Além disso, possui um Centro de Atenção Psicossocial (CAPS) e um Centro de Especialidades Odontológicas (CEO).

O lócus de realização da pesquisa consistiu em uma USF localizada na zona urbana do município de Araripe. De acordo com dados do Sistema de Informação da Atenção Básica (SIAB), esse serviço de saúde possui atualmente uma população adscrita de 3.005 pessoas; dessas, 322 são idosas, o que corresponde a $11 \%$ do total ${ }^{(13)}$. A referida unidade de saúde possui um total de seis idosos acamados, que são cuidados por algum membro da família.
O universo da pesquisa foi composto por seis cuidadores familiares dos idosos adscritos à unidade de saúde supracitada, no entanto, houve duas recusas. Assim, a amostra foi constituída por quatro cuidadores. A inclusão dos participantes obedeceu aos seguintes critérios: a) ser cuidador(a) principal do(a) idoso(a) dependente; b) possuir grau de parentesco de $1^{\circ}$ ou $2^{\circ}$ grau; c) residir com o(a) idoso(a); d) possuir capacidade cognitiva para responder à entrevista.

Durante a pesquisa, empregou-se o critério de "saturação dos dados", já que na pesquisa qualitativa o critério numérico não é prioridade, bem como não há preocupação com generalização $0^{(9)}$.

Para a coleta dos dados, aplicou-se um roteiro de entrevista semiestruturada. Foram investigadas as características sociodemográficas do cuidador: nome, idade, sexo, estado civil, escolaridade, religião, ocupação e grau de parentesco; bem como dados relacionados ao idoso, tais como: idade, sexo, estado civil e doença de base associada à dependência e à restrição ao leito. A questão norteadora para o cuidador foi: como você vivencia o cuidado ao idoso acamado?

O roteiro de entrevista semiestruturada deve desdobrar os vários indicadores considerados essenciais e suficientes em tópicos que contemplem a abrangência das informações esperadas. Os tópicos devem funcionar apenas como lembretes, devendo, na medida do possível, ser memorizados pelo investigador quando está em campo. Servindo de orientação e guia para o andamento da interlocução, o roteiro deve ser construído de forma que permita flexibilidade nas conversas ${ }^{(14)}$.

No intuito de facilitar a captação das informações, utilizou-se um gravador digital para reprodução na íntegra das falas dos sujeitos da pesquisa, a fim de se evitar riscos de interpretações equivocadas. Esse recurso possibilita que o investigador fique atento à fala do entrevistado ${ }^{(9)}$.

A organização dos dados ocorreu por meio do método de análise de conteúdo. O processo de análise de conteúdo pode ser dividido em três fases: pré-análise, exploração dos resultados e interpretação das informações ${ }^{(15)}$. A pré-análise é a própria organização do trabalho. É nela que se faz a escolha do objeto de estudo, bem como a formulação dos objetivos do trabalho. Estando decidido o que estudar, é necessário proceder à constituição do corpus. Corpus nada mais é que o conjunto do material submetido a uma análise. A exploração do material consiste em uma fase longa, com procedimentos de codificação ou enumeração em função de regras previamente formuladas.

Estatísticas simples ou complexas permitem estabelecer um panorama de resultados com base em tabulações ou diagramas, os quais condensam as informações fornecidas 
para a análise. A última fase do processo de análise de conteúdo é a interpretação dos resultados, que se baseia literalmente em interpretar os resultados encontrados. Para isso, é necessário voltar-se atentamente aos marcos teóricos pertinentes à investigação, pois eles permitem o embasamento dentro das perspectivas significativas para o estudo, haja vista fundamentar os resultados encontrados ${ }^{(15)}$.

A partir da análise dos depoimentos dos cuidadores, emergiram três categorias temáticas: o processo de dependência do idoso; dificuldades vivenciadas no dia a dia pelo cuidador; e satisfação com o serviço de saúde no domicílio.

Por se tratar de uma pesquisa com pessoas, foram observados os preceitos éticos conforme a Resolução 466/2012 (Diretrizes e Normas Regulamentadoras de Pesquisas envolvendo Seres humanos) do Conselho Nacional de Saúde - CNS/MS ${ }^{(16)}$.

Este estudo obteve a aprovação do Comitê de Ética em Pesquisa da Universidade Federal do Ceará, sob o parecer número 660.902 enúmero do CAAE24721914.8.0000.5054. Os cuidadores entrevistados preencheram o Termo de Consentimento Livre e Esclarecido previamente à realização da entrevista. Para manter o anonimato, os entrevistados foram chamados por: E1, E2, E3 e E4.

\section{RESULTADOS E DISCUSSÃO}

Aseguir, estão apresentados os dados sociodemográficos dos entrevistados e as informações relacionadas aos idosos para, em seguida, serem apresentadas as categorias temáticas emergidas do estudo.

A pesquisa procedeu com quatro cuidadoras do sexo feminino, com idades entre 41 e 72 anos e parentesco de primeiro grau. Com relação à escolaridade, três eram sem escolaridade e uma cursara o ensino fundamental incompleto.

De modo semelhante, um estudo que traçou o perfil do cuidador familiar do paciente com sequela de Acidente Vascular Encefálico (AVE) também mostrou que a maioria dos entrevistados era do sexo feminino ${ }^{(17)}$.

No tocante ao grau de parentesco, a literatura destaca que o papel de cuidador é deslocado para os filhos quando o cônjuge já é falecido ou não pode assumir essa função. Nesse caso, o cuidar é permeado por uma obrigação moral proveniente de valores impostos pela cultura familiar, a qual considera que os filhos devem cuidar de seus pais como retribuição aos cuidados prestados por estes durante a infância e adolescência daqueles ${ }^{(18)}$.

Com relação à escolaridade, os dados encontrados neste estudo vão de encontro a outro estudo também realizado com cuidadores familiares de idosos, segundo o qual a maioria dos participantes havia estudado de um a quatro anos e poucos eram analfabetos ${ }^{(19)}$.

Quanto ao estado civil, duas eram casadas e as outras duas eram viúvas, mas as quatro cuidadoras possuíam filhos. Sobre religião, todas declararam ser católicas. A profissão de três das entrevistadas era agricultora e a outra era empregada doméstica. $\mathrm{O}$ tempo de cuidado decorrido desde o início da dependência variou de dois a oito anos.

A idade dos idosos variou entre 73 e 92 anos. Quanto ao sexo, três eram mulheres e um homem. Com relação ao estado civil, dois eram casados e os outros dois, viúvos. As doenças de base associadas à dependência e restrição ao leito foram: incontinência urinária e fecal, demência, AVE e neoplasia.

\section{O processo de dependência do idoso}

O processo de dependência do idoso, de acordo com os participantes, mostra que ele ocorreu predominantemente por processos patológicos. Apenas uma entrevistada informou que a dependência do idoso esteve associada a um processo fisiológico. Os depoimentos a seguir refutam essa informação:

"Ele começou a sentir dor na urina [...] aí a gente veio no médico [...] ele disse que tinha que operar [...] está com seis anos que ele está dependente de mim." (E1)

"Ela foi dormir de noite, ai quando se acordou foi conversando [...] deu aquele problema, o derrame! Ai levamos ela para o hospital, eles passaram uns remédios [...] ela esmoreceu, as juntas ficaram duras [...] é o reumatismo também!" (E2)

"[...] de seis anos para cá, o corpo dela foi enfraquecendo [...] já vai fazer oito anos que ela vive doente [...] agora ela está se alimentando por uma sonda, não conseguiu mais nem se alimentar. O médico diz que ela não tem doença, diz que isso é devido ao enfraquecimento, devido à idade, e não sabe por que ela ficou desse jeito, porque ela nunca fez cirurgia, coisa nenhuma." (E4)

Notou-se que o processo de dependência e a consequente restrição ao leito ocorreu como consequência de processos patológicos já citados, como neoplasia, acidente vascular encefálico e demência. No entanto, notou-se também que os fenômenos fisiológicos próprios da velhice também podem levar o indivíduo a tornar-se dependente dos cuidados de outra pessoa.

O AVE conceitua-se como um sinal clínico de rápido desenvolvimento de perturbação focal da função cerebral, de suposta origem vascular e com mais de 24 horas. Possui alta incidência entre os idosos. Anualmente, 15 milhões de pessoas em todo o mundo sofrem um acidente vascular encefálico; destas, cinco milhões morrem e outros 
cinco milhões ficam com algum grau deficiência, gerando incapacidade funcional ${ }^{(20)}$.

Ressalta-se a importância de a ESF investigar o histórico do paciente idoso para compreender os fatores associados à dependência, indicar medidas que podem ser executadas para prevenir possíveis agravos advindos da dependência e subsidiar a análise da evolução do estado de saúde do idoso.

Diante da dependência, o cuidador familiar tornase o principal responsável pelo idoso - não por opção, mas geralmente por obrigação, conforme os depoimentos expostos a seguir:

"Porque não tinha quem cuidasse, aí tinha que ser eu [...] era a filha que tinha. A outra não queria cuidar, disse que não podia. Eu não ia deixar eles desprezados, ai tive que cuidar deles [...]. Os outros faziam assim: chegavam lá, faziam o almoço e iam embora [...] ai dois clientes desse jeito, não tem como ficar dentro de uma casa sem ter uma pessoa para orientar. Tem a hora do remédio, a hora da comida, a hora de tomar um banho, ai a causa de eu ter vindo para cá foi essa." (E1)

"Eu me sinto presa a ela, porque a gente tem que ficar cuidando dela. Tem os remédios, os banhos... mas eu não acho ruim cuidar dela. Eu acho bom, porque ela é minha mãe, eu faço com gosto." (E3)

"Eu me vejo cansada, porque ela depende de tudo, e eu também sou doente da coluna. Aí fica pior para cuidar direito dela." (E4)

Observou-se que o papel de cuidador é assumido pela filha, ou seja, parente de primeiro grau, que vivencia muitas dificuldades, ligadas principalmente à indisponibilidade de outras pessoas da família para auxiliar na realização das tarefas associadas ao cuidado com idoso, fato que ocasiona sobrecarga de trabalho, a qual reflete negativamente na sua qualidade de vida.

Em contrapartida, o sentimento de obrigação dá lugar ao afeto à medida que o cuidado se reveste de um sentimento de amor e retribuição ${ }^{(18)}$.

Historicamente, a mulher sempre foi responsável pelo cuidado, seja da casa ou dos filhos, enquanto o papel do homem era trabalhar fora para garantir o provimento financeiro da família. E apesar de todas as mudanças sociais e na composição familiar, e dos novos papéis assumidos pela mulher, destacando-se a sua maior participação no mercado de trabalho, ainda se espera que a mulher assuma essa função ${ }^{(21)}$.

É papel da equipe de saúde sensibilizar a família do idoso quanto à importância da divisão das tarefas para amenizar a sobrecarga de atividades e proporcionar estabelecimento de uma relação de corresponsabilidade entre os membros da família no tocante ao cuidado com o idoso.

\section{Dificuldades vivenciadas no cotidiano pelo cuidador}

A categoria "dificuldades vivenciadas no cotidiano do cuidador" mostra que os problemas estão relacionados à realização dos cuidados diários, a exemplo da higiene pessoal, alimentação e mudança de decúbito, segundo os testemunhos a seguir:

"Assim, bem cedo pra gente levantar, pra gente banhar ele [...]. Ele não usa fralda, ele só usa o short. A comida dele eu tenho que dar na boca [...]. Eu acho uma dificuldade muito grande [...]. Assim, a dificuldade que tem é porque eu não posso levantar ele só, mas eu tenho que levantar [...]. Olha o tanto de lençol que eu já lavei. Eu já lavei uns vinte lençóis hoje por causa do xixi." (E1)

"Rapaz, enquanto ela está quietinha, tudo bem. Agora, o pior mesmo é a hora de dar banho, porque sozinha eu não resolvo [...]. Tem que depender de outra pessoa, porque ela pesa e eu não posso pegar ela sozinha, colocar na cadeirinha, trazer e banhar." (E3)

Como mencionado nos depoimentos, a higiene pessoal do idoso, principalmente a realização do banho, é a principal dificuldade vivenciada pelo cuidador. Isso ocorre devido ao déficit de locomoção da pessoa cuidada, que exige do cuidador a utilização de uma maior força física para transportar esse indivíduo de um ambiente para outro, bem como para realizar as mudanças de decúbito no leito. Além disso, observa-se também a ausência de um assistente para a realização dessas tarefas, o que acaba gerando uma sobrecarga para o cuidador, o qual muitas vezes também é idoso, dificultando ainda mais o processo de cuidar.

Os cuidadores familiares são aqueles que atendem às necessidades de autocuidado de indivíduos com algum grau de dependência, como alimentação e higiene. Verifica-se também que, na maioria das vezes, o cuidado é realizado por mulheres, filhas ou esposas que residem com o idoso e cuidam dele em tempo integral, sendo quase sempre uma atividade solitária, realizada sem revezamento com outros familiares $^{(22)}$.

O mesmo estudo mostrou que a sobrecarga do cuidador está diretamente relacionada a distúrbios psicoemocionais, ao tempo de dedicação ao cuidado, à falta de informação do cuidador, ao grau de dependência do idoso, à presença de depressão e à incontinência ${ }^{(22)}$.

Diante das dificuldades vivenciadas pelos cuidadores, fica clara a importância de as ESF e o Núcleo de Apoio à Saúde da Família (NASF) prestarem suporte emocional ao cuidador, seja por meio da visita domiciliar, escuta terapêutica e/ou estabelecimento de grupos para compartilhamento de experiências. Ademais, o foco desses profissionais deve contemplar esforços para aquisição da autonomia e independência do idoso, potencializando o autocuidado $^{(23)}$. 


\section{Satisfação com o serviço de saúde no domicílio}

Esta categoria está relacionada à assistência prestada pela ESF no domicilio, de acordo com as inferências que seguem:

"[...] assim, chega o dia da visita [...], ai eles chegam, vão botar o aparelho em pai, olha como está a pressão de pai [...], dizem que está bem, que está bem cuidado. [...] eu peço para eles avisarem quando for para vir, porque às vezes chega e eu estou lavando roupa." (E1)

"Gosto, Ave Maria! Porque às vezes a gente está sentindo uma dor, está com uma tontice, então eles vêm aqui. A gente chama, ai quando dá fé o remédio chega. Ai quando eles veem que não dá certo, manda ir para o hospital lá no posto." (E2)

"É legal, porque tem os dias que eles vêm aqui. Assim, é só uma vez no mês [...]. Se fosse feita de oito em oito dias [...], mas não pode [...]. Mesmo assim a gente se sente feliz, porque eles acompanham sempre, vê como ela está." (E3)

"É bom, porque eles sempre dão força; eles vêm aqui olhar [...]. Quando precisa, levam para o hospital e tudo." (E4)

Ficou evidenciado que a realização das visitas domiciliares é muito bem vista pelas entrevistadas, pois promove a criação de vínculo com a comunidade, tornando a assistência mais eficaz e resolutiva.

Tendo em vista que as pessoas idosas, em condições de fragilidade ou de maior vulnerabilidade, não comparecem com tanta frequência aos serviços de saúde, a modalidade de atenção domiciliária deve ser planejada pela ESF, garantindo o vínculo com o sistema de saúde ${ }^{(24)}$.

A assistência realizada no domicílio pela ESF tem seu foco em ações de orientação quanto ao tratamento medicamentoso, higiene pessoal e alimentação, além da realização de procedimentos básicos, como a aferição da pressão arterial, conforme as citações a seguir:

"Eles orientam que eu dê o remédio dele, perguntam o remédio que ele toma. Se tiver faltando algum remédio, eu digo que está faltando. Se tiver no postinho, eles dão. E se não tiver, eles dão a receita para eu comprar." (E1)

"[...] eles medem a pressão dela e dizem que ela é sadia, porque não tem problema de coração. Só o que eles cobram mais da saúde dela é dos ossos, que tem hora que os ossos estão duros, as pernas não querem ir [...]" (E2)

"Ter cuidado com a sonda, ter sempre o cuidado com a higiene, com a alimentação, que não pode descuidar de nada. Eles vêm medir a pressão, vêm ouvir bem direitinho a pessoa." (E3)

Verificou-se que o trabalho realizado pelas equipes de saúde no domicílio tem como foco o fornecimento de informações relacionadas principalmente à alimentação e ao uso correto de medicações, que são fundamentais para promoção da saúde e prevenção de agravos, como as iatrogenias, comuns nessa fase da vida devido ao uso contínuo de medicações.

De fato, a visita domiciliar configura-se um espaço privilegiado para o contato e o desenvolvimento de atividades educativas com a família e outras pessoas significantes, que são o suporte social aos usuários e contribuem para a manutenção de hábitos saudáveis ${ }^{(25)}$.

\section{CONSIDERAÇÕES FINAIS}

O estudo evidenciou três aspectos importantes: as causas associadas à dependência do idoso; os principais problemas vivenciados no cotidiano dos cuidadores; e a satisfação com o serviço de saúde.

O processo de dependência e a consequente restrição ao leito ocorreu como consequência de processos patológicos, como neoplasia, acidente vascular encefálico e demência. No entanto, notou-se também que os fenômenos fisiológicos próprios da velhice também podem levar o indivíduo a tornar-se dependente dos cuidadores.

Quanto aos problemas enfrentados pelo cuidador, ressalta-se a necessidade de um envolvimento maior da família quanto à divisão de tarefas, haja vista que a centralização do trabalho gera sobrecarga e redução da qualidade de vida do cuidador e, consequentemente, afeta o cuidado com o idoso.

A satisfação com o serviço de saúde relaciona-se diretamente com o vínculo que se institui entre o serviço de saúde e a família no ambiente domiciliar. O trabalho em equipe no contexto domiciliar é fundamental, pois permite um atendimento complementar e integral para o binômio idoso/cuidador.

Nessa perspectiva, faz-se necessário um maior investimento no cuidado domiciliar, a exemplo da expansão do Serviço de Atendimento Domiciliar (SAD), pois permite um atendimento complementar e integral, contando com uma equipe multidisciplinar, que trabalha tanto na prevenção de agravos quanto na promoção da saúde dos acamados em domicílio.

\section{REFERÊNCIAS}

1. Silva MRF, Yazbek MC. Proteção social aos idosos: concepções, diretrizes e reconhecimento de direitos na América Latina e no Brasil. R. Katálysis. 2014;17(1):102-10.

2. Belmonte LM, Lima IAX, Belmonte LAO, Gonçalves VP, Conrado JC, Ferreira DA. Força de preensão manual 
de idosos participantes de grupos de convivência. Rev Bras Promoç Saúde. 2014;27(1):85-91.

3. Instituto Brasileiro de Geografia e Estatística - IBGE. Censo demográfico 2010. Rio de Janeiro; 2010 [acesso em2015 Jan 28]. Disponívelem: http://www.censo2010. ibge.gov.br/sinopse/index.php?dados $=12 \& u f=00$.

4. Bezerra FC, Almeida MI, Nóbrega-Therrien S. Estudos sobre envelhecimento no brasil: revisão bibliográfica. Rev Bras Geriatr Gerontol. 2012;15(1):155-67.

5. Veras RP. Estratégias para o enfrentamento das doenças crônicas: um modelo em que todos ganham. Rev Bras Geriatr Gerontol. 2011;14(4):779-86.

6. Moraes EN. Atenção à saúde do idoso: aspectos conceituais. Brasília: Organização Pan-Americana da Saúde; 2012.

7. Brasil. Constituição da República Federativa do Brasil: texto constitucional promulgado em 5 de outubro de 1988, com as alterações adotadas pelas emendas Constitucionais nos 1/1992 a 68/2011, pelo Decreto legislativo $\mathrm{n}^{\circ} 186 / 2008$ e pelas emendas Constitucionais de Revisão nos 1 a 6/1994. 35ª ed. Brasília: Câmara dos Deputados; 2012.

8. Floriano LA, Azevedo RCS, Reiners AAO, Sudré MRS. Cuidado realizado pelo cuidador familiar ao idoso dependente, em domicílio, no contexto da estratégia de saúde da família. Texto \& Contexto Enferm. 2012;21(3):543-8.

9. Marconi MA, Lakatos EM. Técnicas de pesquisa: planejamento e execução de pesquisas, amostragens e técnicas de pesquisa, elaboração, análise e interpretação de dados. São Paulo: Atlas; 2011.

10. Pereira JM. Mais sobre manual de metodologia da pesquisa científica. $3^{\text {a }}$ ed. São Paulo: Atlas; 2012.

11. Kahlmeyer-Mertens RS. Como elaborar projetos de pesquisa: linguagem e método. Rio de Janeiro: FGV; 2014.

12. Governo do Estado do Ceará. Apresenta informações sobre os municípios cearenses. 2014 [acesso em 2015 Jan 26]. Disponível em: http://www.ceara.gov.br/ municipios-cearenses/780-municipios-com-a-letraa\#munic-pio-araripe

13. Ministério da Saúde (BR), Secretaria de Atenção à Saúde, Departamento de Atenção Básica. Sistema de Informação da Atenção Básica - SIAB: indicadores 2014. Brasília: Ministério da Saúde; 2014.
14. Canzonieri AM. Metodologia da pesquisa qualitativa na saúde. Petrópolis: Vozes; 2015.

15. Bardin L. Análise de conteúdo. São Paulo: Edições 70; 2011.

16. Brasil (BR). Resolução $\mathrm{n}^{\circ} 466$, de 12 e dezembro de 2012. Brasília: Conselho Nacional de Pesquisa; 2012.

17. Pereira RA, Santos EB, Fhon JRS, Marques S, Rodrigues RAP. Sobrecarga dos cuidadores de idosos com acidente vascular cerebral. Rev Esc Enferm USP. 2013;47(1):185-92.

18. Vieira L, Nobre JRS, Bastos CCBC, Tavares KO. Cuidar de um familiar idoso dependente no domicílio: reflexões para os profissionais da saúde. Rev Bras Geriatr Gerontol. 2012;15(2):255-63.

19. Santos AA, Pavarini SCL. Funcionalidade familiar de idosos com alterações cognitivas: a percepção do cuidador. Rev Esc Enferm USP. 2012;46(5):1141-7.

20. Santos NMF, Tavares DMS. Correlação entre qualidade de vida e morbidade do cuidador de idoso com acidente vascular encefálico. Rev Esc Enferm USP. 2012;46(4):960-6.

21. Gratao ACM, Vendrúscolo TRP, Talmelli LFS, Figueiredo LC, Santos JLF, Partezani RA. Sobrecarga e desconforto emocional em cuidadores de idosos. Texto \& Contexto Enferm. 2012;21(2):304-12.

22. Oliveira DC, D’Elboux MJ. Estudos nacionais sobre cuidadores familiares de idosos: revisão integrativa. Rev Bras Enferm. 2012;65(5):829-38.

23. Araújo I, Paúl C, Martins M. Viver com mais idade em contexto familiar: dependência no auto cuidado. Rev Esc Enferm USP. 2011;45(4):869-75.

24. Castro BMMM, Telles JL. O Cuidado do Idoso no contexto familiar: percepção da equipe de saúde da família. Rev Bras Geriatr Gerontol. 2010;13(3):34960 .

25. Ribeiro AG, Cotta RMM, Silva LS, Ribeiro SMR, Dias CMGC, Mitre SM. Hipertensão arterial e orientação domiciliar: o papel estratégico da saúde da família. Rev Nutr. 2012;25(2):271-82.

\section{Endereço para correspondência:}

Ticiano Magalhães Dantas

Rua Waldir Silva, 50 apto 1 Bairro: Sossego

CEP: 63.107-280 - Crato - CE - Brasil

E-mail: ticianotmd@hotmail.com 